TENDENCIAS

Revista de la Facultad de Ciencias

Económicas y Administrativas.

Universidad de Nariño

ISSN-E 2539-0554

Vol. XXII No. 1 - 1er Semestre 2021,

Enero-Junio - Páginas 95-119

\title{
EMPRENDIMIENTO Y DISEÑO: CARACTERIZACIÓN DE LAS INICIATIVAS EMPRENDEDORAS DE LOS DISEÑADORES INDUSTRIALES ${ }^{1}$
}

ENTREPRENEURSHIP AND DESIGN: CHARACTERIZATION OF ENTREPRENEURIAL INITIATIVES OF INDUSTRIAL DESIGNERS

\section{EMPREENDEDORISMO E DESIGN: CARACTERIZAÇÃO DAS INICIATIVAS EMPREENDEDORAS DOS DESIGNERS INDUSTRIAIS}

Maria Cristina Ascuntar Rivera; Francisco Rafael Ayala Gallardo

Magíster en Diseño y Creación Interactiva, Universidad de Caldas. Docente Investigadora Departamento de Diseño, Universidad de Nariño. ORCiD: 0000-0003-2671-3909. E-mail: cristinascuntar@udenar.edu.co, Colombia.

Magíster en Diseño de Experiencia de Usuario, Universidad Internacional de la Rioja. Docente Investigador Departamento de Diseño, Universidad de Nariño. ORCiD: 0000-0003-4090-1631. Email: franciscoayala@udenar.edu.co, Colombia.

Recibido: 5 de junio de 2020

Aprobado: 15 de octubre de 2020

DOI: https://doi.org/10.22267/rtend.202102.156

\footnotetext{
${ }^{1}$ Este artículo es resultado del proyecto de investigación titulado: Diagnóstico de las iniciativas emprendedoras de los egresados del programa de Diseño Industrial de la Universidad de Nariño, aprobado y financiado por la Vicerrectoría de Investigaciones, Posgrados y Relaciones Internacionales VIPRI de la Universidad de Nariño, mediante Acuerdo $\mathrm{N}^{\circ} 135$ del 19 septiembre de 2017. El proyecto fue presentado en la convocatoria anual para conformar un banco de proyectos docentes de Investigación Científica, Desarrollo Tecnológico, Innovación y Creación Artística, elegibles para ser financiados por la VIPRI 2017.
} 


\title{
Resumen
}

En el artículo se exponen los resultados de la caracterización de las iniciativas emprendedoras, desarrolladas por egresados del programa de Diseño Industrial de la Universidad de Nariño. El propósito del estudio fue indagar la trayectoria de los trabajos de grado en la modalidad de creación de empresas, que se establece en la carrera de Diseño Industrial. Desde la perspectiva del diagnóstico, el proyecto planteó el reconocimiento, análisis y evaluación de la situación de los egresados quienes obtuvieron su titulación bajo esta modalidad. Lo anterior, condujo a describir características de los proyectos e identificar las percepciones de los egresados sobre la formación académica, relacionada con el desarrollo de emprendimientos en diseño. Para tal propósito, la metodología se realizó bajo dos instancias que fueron la recopilación de información a partir fuentes primarias y secundarias, y posteriormente, el análisis de datos y valoración del estado actual de estos proyectos, que permitió hacer evidente la necesidad de generar escenarios investigativos, curriculares y pragmáticos, que aporten al fomento y promoción del emprendimiento en el entorno académico del área de diseño.

Palabras clave: creación de empresa; diseño; emprendedor; idea de negocio.

JEL: A13; L20; M13; M19

\begin{abstract}
The article presents the results of the characterization of entrepreneurial initiatives, developed by graduates of the Industrial Design program of the University of Nariño. The purpose of the study was to investigate the trajectory of the degree works in the modality of creation of enterprises, which is established in the career of Industrial Design. From the perspective of the diagnosis, the project proposed the recognition, analysis and evaluation of the situation of the graduates who obtained their degree under this modality. This led to the description of the characteristics of the projects and the identification of the perceptions of the graduates about the academic formation, related to the development of design enterprises. For this purpose, the methodology was carried out under two instances that were the collection of information from primary and secondary sources, and subsequently, data analysis and assessment of the current status of these projects, which made evident the need to generate research scenarios, curricular and pragmatic, which contribute to the development and promotion of entrepreneurship in the academic environment of the design area.
\end{abstract}


Keywords: business start-up; design; entrepreneurship; business idea.

JEL: A13; L20; M13; M19

\section{Resumo}

O artigo apresenta os resultados da caracterização das iniciativas empresariais, desenvolvidas pelos graduados do programa de Desenho Industrial da Universidade de Nariño. O objetivo do estudo foi investigar a trajetória dos trabalhos de graduação na modalidade de criação de empresas, que se estabelece na carreira de Desenho Industrial. A partir da perspectiva do diagnóstico, o projeto propôs o reconhecimento, análise e avaliação da situação dos graduados que obtiveram sua graduação sob esta modalidade. Isto levou à descrição das características dos projetos e à identificação das percepções dos graduados sobre a formação acadêmica, relacionadas ao desenvolvimento de empreendimentos de design. Para este fim, a metodologia foi realizada sob duas instâncias, que foram a coleta de informações de fontes primárias e secundárias e, posteriormente, a análise de dados e avaliação da situação atual destes projetos, o que tornou evidente a necessidade de gerar cenários de pesquisa, curriculares e pragmáticos, que contribuam para o desenvolvimento e promoção do empreendedorismo no ambiente acadêmico da área de design.

Palavras-chave: criação de empresas; design; empreendedorismo; idéia de negócios.

JEL: A13; L20; M13; M19

\section{Introducción}

La cultura del emprendimiento se ha posicionado como una tendencia a escala mundial y durante el último decenio, se ha consolidado no solo en una oportunidad para el desarrollo humano y económico de los individuos, sino también como estrategia efectiva para el crecimiento de las economías desarrolladas, emergentes y en vía de desarrollo. Estudiosos del tema como María Minniti (2012), sostienen que se evidencia un cambio del paradigma del capitalismo administrativo al capitalis mo emprendedor, situación que implica transformaciones en la manera de pensar y hacer las cosas, particularmente en la forma de concebir al emprendedor y donde la noción de emprendimiento ocupa un lugar central como motor del desarrollo económico. 
Dentro de este escenario prometedor del emprendimiento, los gobiernos alrededor del mundo admiten la necesidad de dar respuesta a los intereses, necesidades y expectativas de los emprendedores desde entidades del sector público y privado, que han aceptado el reto a esa demanda, con la implementación de estrategias para el fomento, promoción y apoyo en todos los niveles de desarrollo, desde la cultura del emprendimiento, la iniciativa emprendedora, la generación y valoración de idea, modelo y plan de negocios, hasta su puesta en marcha con la financiación del capital de riesgo o semilla (Schnarch, 2014). Derivado de lo anterior, el proceso de emprendimiento se ha transformado en un entorno extenso, complejo y dotado de distintas variables, que ha llevado a constituir los denominados Ecosistemas Emprendedores, como un modelo de cadena de valor en procura de alcanzar los propósitos del emprendimiento. Según lo expuesto por Arenal, Armuña, Ramos, y Feijóo (2018), el concepto de ecosistema emprendedor surge para representar un fenómeno que se desarrolla en un entorno definido, haciendo hincapié en las interacciones que suceden entre los diversos agentes que cohabitan en un determinado lugar.

La importancia del emprendimiento no es solo percibida por las entidades y organizaciones gubernamentales, puesto que los ciudadanos también le atribuyen trascendencia a la actividad emprendedora en el desarrollo del individuo y en su aporte a la transformación de la sociedad; lo anterior se correlaciona con lo expuesto por Uribe y Reinoso (2013) infiriendo que "para el emprendimiento existen factores de índole personal o individual y factores relacionados con el contexto, que entendidos bajo un enfoque sistémico, conforman el ecosistema emprendedor” (p. 15). Así mismo, cabe resaltar que en Colombia los esfuerzos para el fomento y promoción del emprendimiento, se consolidan con la Ley 1014 de 2006 de Fomento a la Cultura del Emprendimiento, promovida por el Ministerio de Comercio, Industria y Turismo, con miras a facilitar las condiciones para el emprendimiento y el desarrollo de su dimensión local, definiendo además los objetivos estratégicos, las entidades y actores responsables del proceso, los mecanismos y recursos para la financiación.

En el ámbito regional, conviene hacer mención del Ecosistema de Emprendimiento Región Suroccidente, que está compuesto por dos nodos con sus respectivas ciudades. Según el Mapeo e Infografía de Ecosistemas Regionales del Emprendimiento en Colombia (iNNpulsa Colombia, 2016), la región sur occidente está conformada por el nodo uno departamento del Valle del Cauca: Cali, 
Palmira, Yumbo y Jamundí, Cauca con la ciudad de Popayán y el nodo dos correspondiente a la ciudad de Pasto; este último por ser capital del departamento de Nariño, agrupa iniciativas de los municipios de Ipiales y Tumaco. Con base al mapeo, para el año 2016 el nodo dos de la ciudad de Pasto, soporta diez y ocho (18) startup, cinco (5) instituciones del sector productivo, dos (2) instituciones de apoyo financiero, tres (3) otras organizaciones y catorce (14) instituciones de apoyo académico; en este último grupo se encuentra la Universidad de Nariño, como institución pública de educación superior.

Con relación al contexto académico objeto de estudio, según se detalla en el Proyecto Educativo del Programa de Diseño Industrial PEP (Departamento de Diseño, 2013), uno de los fundamentos para la creación de este programa de pregrado, fueron los bajos índices de creación de empresa a nivel local y regional, como también la ausencia de una cultura emprendedora con características de creatividad e innovación. En consecuencia, según la vigente Reglamentación de Trabajos de Grado (Departamento de Diseño, 2006), el programa de Diseño Industrial cuenta con cinco modalidades de grado que son: proyecto de diseño industrial, proyecto de creación de empresas, proyecto de investigación-monografía, pasantía y diplomado. Siendo el tema de interés de esta investigación la modalidad de creación de empresas, en principio la evidencia empírica llevó a realizar indagaciones previas para determinar la situación de esta modalidad y la baja tasa de participación con respecto a las otras opciones. Por tanto, fue necesario realizar esta investigación de diagnóstico y caracterización, para determinar el estado de las iniciativas emprendedoras de los egresados del programa de Diseño Industrial de la Universidad de Nariño.

\section{Referentes teóricos}

\section{Panorama general}

El interés global por el emprendimiento se ha hecho evidente en diversos escenarios tales como el Foro Económico Mundial de 2015, donde fue presentado el estudio titulado Apalancando la ambición y la innovación emprendedora citado por Cuevas (2016), en donde se refleja que Colombia ocupa el cuarto lugar en el listado de países con mayor número de emprendimientos innovadores en fase inicial y donde el reto, es seguir impulsando estos nuevos negocios. Sin embargo, GEM Colombia en un estudio realizado sobre de la actividad empresarial del año 2017 (Gómez et al., 2019), planteó una cifra donde el país se ubicaba como la sexta economía con la tasa de empresarios establecidos más 
baja en América Latina y el Caribe (4,9\%), indicador que demuestra cómo una parte importante de la población adulta en el país, no está logrando consolidar sus empresas.

Otros estudios como los del Banco de Desarrollo para Latinoamérica de Sanguinetti et al. (2013), confirman que el emprendimiento es un sector de provecho para grandes organizaciones gubernamentales, datos que son el resultado de indagar en las iniciativas emprendedoras que se han llevado a cabo en distintos países de la región, cuyo crecimiento se ha hecho perceptible hasta un $80 \%$. En la misma medida, el panorama del emprendimiento en Latinoamérica se ha extendido y ha sido registrado a través de diversas publicaciones tales como el documento de Lederman et al. (2014) del Banco Mundial titulado El emprendimiento en América Latina: muchas empresas y poca innovación, donde se ratifica la necesidad de consolidar una cultura emprendedora basada en la evolución social y económica. Así mismo, la publicación Entrepreneurship in Latin America: A step up the social ladder (Lora y Castellani, 2014), una coproducción del Banco Interamericano de Desarrollo BID y del Banco Mundial, pone de manifiesto la relevancia de crear espacios gubernamentales que propendan por el fomento de la cultura emprendedora, en las comunidades con potencial para desarrollar proyectos basados en la creación de empresas.

\section{El proceso de emprendimiento}

El término emprendimiento está directamente relacionado con la acción de emprender. Shapero y Sokol (1982), plantean un esquema de tres fases donde se enmarca la actividad emprendedora como gestión del cambio radical e intermitente, o renovación estratégica. La primera, es una fase previa donde suceden una serie de acontecimientos, bien sea positivos o negativos, que inducirán al emprendedor a constituir su empresa. Subsecuentemente, en la fase de deseo existe una serie de circunstancias, entre las que cabe anotar la formación del emprendedor, la familia, la cultura y amistades, factores que transformarán al posible emprendedor en un emprendedor potencial. En tercera instancia, la fase de acción sucede bajo una serie de condiciones donde el individuo finalmente se decidirá a crear su empresa; dichas condiciones podrían relacionarse con la existencia de recursos financieros, humanos y técnicos, entre otros.

Con base en la Ley 1014 de 2006 que se constituye en el documento de la política pública de emprendimiento, define este término como un conjunto de personas, variables y factores que 
intervienen en el proceso de crear una empresa. Se instaura en una manera de pensar y actuar orientada hacia la creación de riqueza y también es una forma de razonar y actuar centrada en las oportunidades, planteada con visión global y llevada a cabo mediante un liderazgo equilibrado y la gestión de un riesgo calculado, cuyo resultado es la creación de valor que beneficia a la empresa, la economía y la sociedad. De acuerdo con Uribe y Reinoso (2013), es importante reconocer el concepto de emprendimiento desde la perspectiva de la formación, donde se concibe como un conjunto de capacidades que provienen de la actitud de la persona para visualizar oportunidades de su contexto, lo cual requiere una visión holística, para transformarlas en ideas innovadoras sustentadas en su creatividad y generar proyectos productivos en distintos campos, para beneficio propio y de su entorno. En este aparte, también es necesario considerar las formas en la cuales se exterioriza el emprendimiento, que según exponen Urbano y Toledano (2008), no son únicamente aquellas actividades emprendedoras desarrolladas por una persona como iniciativa propia a partir de una idea de negocio, puesto que la franquicia, la empresa familiar, la empresa social y los proyectos emprendedores en empresas ya establecidas o intraemprendimiento, hacen parte de las manifestaciones de este ecosistema.

\section{El emprendedor}

En lo concerniente al panorama del emprendimiento, es perentorio destacar la presencia del emprendedor, cuyo rol, características, habilidades, destrezas y competencias, son la base de todas las dinámicas que se suscitan en cualquier contexto de la actividad emprendedora. Etimológicamente, Schnark (2014) menciona que el término emprendedor proviene del vocablo francés entrepreneur y tal vez su uso más antiguo se registra en la historia francesa en el siglo XVII y hacía referencia a personas que se comprometían a conducir expediciones militares. Cabe resaltar que ya en siglo XVIII, el economista Richard Cantillon (como se cita en Pulido et al., 2017), manifiesta el interés de acuñar el término y definición de emprendedor, como un individuo apto para asumir riesgo económico y crear empresa. Finalmente, como corolario de esta breve reseña de definiciones, Peter Drucker (1994) gran pensador y filósofo de la administración moderna, con una visión contemporánea cataloga al emprendedor como el resultado de una categoría de individuo o característica exclusiva pero no de personalidad. Considera que el emprendedor es un innovador que visualiza el cambio como práctica saludable y lo aprovecha en su beneficio. Así mismo, destaca que ese rol no se limita al ámbito económico, sino que se aplica a todas las actividades humanas. 


\section{Política pública para fomento y promoción del emprendimiento}

El devenir de la política pública colombiana relacionada con la creación de empresas y el emprendimiento se correlaciona con las realidades sociales, económicas y políticas del país en el siglo XX, influenciadas desde la depresión de la posguerra, la desindustrialización y transición a la política Pyme, hasta las estrategias actuales para el fomento del emprendimiento. Según lo expuesto por Tarapuez et al. (2013), los primeros indicios de la política pública para el emprendimiento en Colombia se vislumbran en el año 2002, cuando dos funcionarios del Gobierno Nacional (con amplia experiencia en el sector empresarial), elevaron el emprendimiento a condición de problema público. Esto provocó generar conciencia en quienes tienen la responsabilidad de formular políticas públicas, para tomar acciones que fomenten y apoyen la actividad emprendedora en los colombianos.

La priorización y legalización del fenómeno de emprendimiento, finalmente se consolida en Colombia con la Ley 1014 de 2006 de Fomento a la Cultura del Emprendimiento, promovida por el Ministerio de Comercio, Industria y Turismo. Según se estipula en esta ley, el papel del Estado en el fomento del emprendimiento se estructura en la promoción de la alianza público-privada académica, como también en facilitar las condiciones y desarrollar la dimensión local del emprendimiento. Cabe considerar también, que la máxima expresión del compromiso del Estado colombiano se evidencia en la expedición de la Política Nacional de Desarrollo Productivo, consignada en el documento CONPES 3866 de 2016 (Departamento Nacional de Planeación [DNP], 2016), donde se establece el papel fundamental que realizan el emprendimiento y la innovación en el desarrollo socioeconómico del país.

Después de esta breve indagación sobre políticas públicas relacionadas con el emprendimiento, cabe destacar algunas reglamentaciones como la Ley 344 de 1996 relacionada con el gasto público, donde se establecen algunas normas correspondientes a la creación de empresas y que en el Artículo 16 trata sobre el acceso a los recursos para proyectos de incubadoras asociadas al SENA. En otra instancia, está la Ley 590 de 2000, por la cual se dictan disposiciones para promover el desarrollo de las micro, pequeñas y medianas empresas, conocida como la Ley MIPYME. Por último, está la Ley 789 de 2002 que en el Artículo 40 normaliza la creación del Fondo Emprender y se dictan normas para apoyar el empleo y ampliar la protección social, que además provocan la modificación de algunos artículos del Código Sustantivo del Trabajo. 


\section{Emprendimiento y academia}

Considerando las potencialidades y valores del emprendimiento, a partir de la primera década del siglo XXI, las instituciones de educación superior iniciaron procesos que permitieran articular la enseñanza con la cultura emprendedora. Respecto a la influencia de la educación superior en el emprendimie nto en Colombia, Urrego y Quiroga (2018) retoman la definición del Ministerio de Educación Nacional en referencia a que la Educación Superior es un proceso de formación constante, personal, cultural y social, fundamentado en la concepción integral de la persona humana y su dignidad, sus derechos y sus deberes. Desde otra perspectiva, Rangel (como se cita en Urrego y Quiroga, 2018) sustenta que las universidades son organizaciones que tienen un papel crucial respecto a la divulgación y transmisión de conocimiento, hecho que las convierte en estructuras relevantes para la economía de un país, por cuanto coadyuvan aportando factores diferenciadores que provocan ventajas competitivas y la sostenibilidad del entorno.

Dentro de este marco, ha de considerarse que la educación en emprendimiento tiene su génesis en el núcleo familiar y son precisamente las empresas familiares, que han heredado tradiciones y prácticas en la creación de empresa, la prueba de los fundamentos de la actividad emprendedora. Posteriormente, este ejercicio se transfiere a los niveles de básica primaria, básica secundaria y media vocacional, cuyas dinámicas se exhiben por medio de las cátedras de emprendimiento implementadas en los currículos y diversos escenarios de divulgación ejemplificados con las ferias. Con las anteriores premisas, es útil agregar que las universidades representan un eslabón importante para el desarrollo del emprendimiento a través de distintas estrategias como las sugeridas por Mazacón et al. (2019), que se constituyen en "planificación, flexibilidad, autoconfianza, responsabilidad, trabajo colaborativo, búsqueda de soluciones, capacidad de persuasión, asertividad, talento creativo, equilibrio entre riesgo y prudencia” (p. 15).

El debate sobre la educación en emprendimiento está abierto desde los campos de la pedagogía, la didáctica y el currículo. Sin embargo, cabe hacer hincapié en la importancia de propender por la práctica de la investigación, que favorezca el desarrollo de actitudes, habilidades y saberes frente a la realidad, donde el docente motiva la indagación e interacción total con el entorno (Pulido et al., 2017). Paralelamente, Alvarado y Rivera (2011) sugieren que el currículo para el emprendimiento representa 
un fenómeno que se construye en la cotidianidad del proceso de enseñanza - aprendizaje y así mismo, debe ser capaz de impulsar un proyecto de formación que se concreta llevándolo a la práctica.

Por lo expuesto anteriormente, es oportuno sugerir que tanto la cultura, las dinámicas pedagógicas, la propuesta educativa, la formación de docentes entre otras variables, deben considerar los modelos de procesos de emprendimiento propuestos por Schnarch (2014) y en la misma línea, reconocer y potenciar el modelo colombiano de Ecosistemas Regionales Emprendedores ERE, que según se describe en el documento Mapeo y caracterización del ecosistema de emprendimiento en Colombia, con énfasis en la localización de las start ups (iNNpulsa Colombia, 2016), se agrupan en cinco instituciones que son: la plataforma regional de emprendimiento, las instituciones de apoyo académico, las instituciones de apoyo financiero, el sector económico y productivo y otras organizaciones de apoyo transversales al proceso de emprendimiento.

\section{Creatividad, emprendimiento y diseño}

El diseño industrial es una profesión relativamente joven que cuenta con un siglo de existencia, desde que se sentaron las bases de la escuela alemana Bauhaus en el año 1919 y cuya razón de ser en principio, fue reconciliar las artes con la técnica, suscitando la premisa en que la forma sigue a la función. Sin embargo, la perspectiva de esta disciplina de carácter proyectual ha cambiado notablemente asumiendo congruentemente la responsabilidad de contribuir al desarrollo socioeconómico, político y cultural de una región. En este sentido, la definición de diseño de la Asociación Mundial del Diseño (World Design Organization [WOD], 2020), en uno de sus apartes sustenta que el diseño industrial ofrece una forma más optimista de mirar el futuro, al reformular los problemas como oportunidades, vinculando la innovación, la tecnología, la investigación, los negocios y los clientes, para proporcionar nuevos valores y ventajas competitivas en las esferas económica, social y ambiental. En esta línea de argumentación, Manuel Lecuona (2006) sustenta que el diseño no debería considerarse de manera aislada y puntual, haciendo referencia únicamente a los aspectos formales de un producto; contrario a lo anterior, el diseño debe ser visto como proceso multidisciplinar, donde intervienen varios especialistas de áreas distintas de la empresa, y donde hay una participación activa del diseñador que no se limita al diseño del objeto. 
Con relación a las bases del diseño, es útil reconocer que su andamiaje principal se sustenta en la creatividad, que se considera la facultad de crear o la capacidad de creación. Respecto a este término y sin profundizar en su definición ontológica, se puede abordar como un proceso cuyo fin es facilitar la resolución de problemas y la toma de decisiones en una organización, provocando que ésta sea más abierta y receptiva para el cambio, donde se evidencia la creatividad como un recurso económico prioritario (Correa et al., 2019). Desde una perspectiva similar, en el Manual de Oslo (OCDE y EUROSTAT, 2006) se establece el concepto de innovación así:

Una innovación es la introducción de un nuevo, o significativamente mejorado, producto (bien o servicio), de un proceso, de un nuevo método de comercialización o de un nuevo método organizativo, en las prácticas internas de la empresa, la organización del lugar de trabajo o las relaciones exteriores. (p. 56)

Con una mirada adicional, es necesario considerar las cavilaciones de Suárez (2017), quien propone que la creatividad y la innovación conjugadas en la investigación y desarrollo I+D, conlleva a que las universidades y sus programas, comprometan esfuerzos para desarrollar pesquisas basadas en la gestión del emprendimiento.

Para culminar esta breve reflexión, conviene mencionar que, en el programa de Diseño Industrial de la Universidad de Nariño se ha observado un compromiso recurrente por fomentar y promover una serie de competencias comunicacionales, investigativas, socio humanísticas, técnicas, adminis trativas y de gestión. La anterior afirmación se soporta en el Proyecto Educativo del Programa de Diseño Industrial PEP (Departamento de Diseño, 2013), que las describe en el marco de la gerencia y liderazgo del diseño como "la capacidad para emprender, desarrollar, optimizar, motivar, organizar, dirigir o coordinar los recursos y los factores del Diseño Industrial” (p. 43). Además, en el plan de estudios, se establecen las asignaturas teóricas y prácticas con dos componentes esenciales en la formación del diseñador que son la creatividad y la innovación, conocimiento y práctica vital para el génesis, desarrollo y éxito de un emprendimiento. 


\section{Metodología}

Respecto a la intención de esta investigación, los objetivos planteados en el proyecto fueron describir las características de las iniciativas emprendedoras y conocer las percepciones de los egresados del programa de Diseño Industrial de la Universidad de Nariño, quienes obtuvieron su titulación bajo la modalidad de grado en creación de empresa. Lo anterior se realizó en procura de adecuar los espacios de formación relacionados con la administración y gestión en diseño, como también exponer las cualidades, capacidades y perfiles de los asesores de trabajo de grado para esta modalidad. En correspondencia con los objetivos trazados, el proyecto se abordó desde un enfoque mixto que se ajusta con las necesidades de indagación, asumiendo un proceso inductivo - deductivo que reside en la experiencia y la observación (Hurtado, 2010). El enfoque cuantitativo se configura en la descripción de datos consolidados y análisis de información, a partir de referencias de los trabajos de grado de todas modalidades del programa. En otra instancia, el enfoque cualitativo permite la interpretación de datos, con la aplicación de métodos y técnicas de indagación de corte observacional, afianzado por métodos subjetivos, en el marco del diseño centrado en el usuario.

En cuanto al alcance de la investigación, es perceptual exploratorio porque implica una aproximac ión inicial al evento, puesto que, aunque hay investigaciones previas de corte aprehensivo, compresivo e integrativo, no hay referentes particulares de estudio para el caso específico referido a los actores y el contexto particular de los diseñadores industriales quienes se titularon con creación de empresa. Dicho esto, el estudio del fenómeno se aborda frente a un diagnóstico que pretende describir las características, propiedades y tendencias de los proyectos de grado de la modalidad de creación de empresas, en correlación con la totalidad de los proyectos para optar el título de diseñador industrial. El contexto de la investigación es la ciudad de Pasto, capital del Departamento de Nariño en el suroccidente colombiano, puntualmente los egresados de Educación Superior de pregrado del programa de Diseño Industrial, de la Facultad de Artes de la Universidad de Nariño, durante en el período comprendido desde el año 2000 a 2018, es decir, un espacio de observación de 18 años correspondiente a la creación del programa y a la reglamentación de la modalidad de creación de empresas, en el año 2006. La investigación se enmarca entre un universo de 456 proyectos de trabajo de grado de las modalidades de desarrollo de producto, monografía, diplomado, pasantía y creación de empresas. 
En concordancia con lo expuesto anteriormente, fue necesario el uso de técnicas de investigación que parten de la revisión documental de información cuantitativa de corte numérico y nominal, de la base de datos del programa de Diseño Industrial entre los años 2000 a 2018. Esta revisión incluye artículos de investigaciones sobre los temas de emprendimiento asociados a la educación superior en los aspectos de desarrollo humano, procedimentales y del sistema emprendedor; libros especializados sobre currículo, pedagogía y didácticas para el emprendimiento, con el propósito de establecer un panorama y horizonte que permita una valoración en el entorno de los proyectos de la modalidad con respecto a otras realidades. Fue relevante la revisión de documentos de mapeos y caracterización de los sistemas emprendedores, que brinda un referente con el contexto regional y local para la valoración de los proyectos de grado; así mismo, fueron sustanciales los documentos de los proyectos de trabajo de grado de la modalidad de creación de empresas. Otras técnicas empleadas fueron la entrevista exploratoria y estructurada de corte etnográfico, con el fin de indagar sobre las percepciones, experiencias y comportamientos de los estudiantes y egresados relacionados con la concepción y proceso de su trabajo de grado.

\section{Discusión}

La investigación abordó en una primera fase, el estudio de la información cuantitativa disponible en las actas de trabajo de grado del programa de Diseño Industrial, entre los años de 2000 a 2018. En estos documentos que reposan en el archivo del Departamento de Diseño, se recabó información sobre fechas, modalidades de grado, títulos de los trabajos, monografías y proyectos, datos de estudiantes, asesores y la correspondiente calificación. Los datos se trataron de forma descriptiva, agrupando los siguientes ítems: histórico de trabajos de grado del programa de diseño industrial, modalidades de trabajo de grado, género de los estudiantes, trabajos de grado modalidad creación de empresas con sus respectivos datos de participantes, género, enfoque de diseño y calificación. En una segunda instancia, se abordó el análisis de información cualitativa, obtenida de las fuentes primarias que corresponden a los egresados titulados en la modalidad creación de empresas, con quienes se aplicó una entrevista semi-estructurada. También cabe adicionar, que se obtuvieron datos importantes de análisis derivados de procesos de autoevaluación y acreditación desarrollados en el Departamento de Diseño. 


\section{Trabajos de grado programa de Diseño Industrial}

Dentro del periodo de observación comprendido desde el año 2000 hasta el 2018, se han planteado, desarrollado y culminado 201 trabajos de grado de 293 egresados del programa de Diseño Industrial. Cabe anotar, que con la Reglamentación de Trabajos de Grado (Departamento de Diseño, 2006) vigente desde el año 2006, se incrementó el número de proyectos de grado que, en correspondencia, también permitió la culminación de los estudios de pregrado a un mayor número de egresados que obtuvieron titulación exitosa. Esta situación ha sido favorable, puesto que se han logrado reducir los índices de egresados quienes por distintas circunstancias no habían alcanzado la finalización de su carrera profesional. Así mismo, la apertura de nuevas modalidades de grado distintas al proyecto de diseño industrial ha permitido visualizar otras perspectivas profesionales y laborales como lo expresan los egresados que fueron entrevistados, quienes consideran propicio que las posibilidades para obtener el título de diseñadores industriales estén directamente correlacionadas con las oportunidades y necesidades socioeconómicas del contexto local y regional.

\section{Modalidades de trabajo de grado}

Con relación a las modalidades de grado del programa de Diseño Industrial, hay una totalidad 201 proyectos desde el año 2000 hasta el 2018, cifra que representa el 100\% (Figura 1), de los cuales el $51 \%$ corresponden al proyecto de diseño industrial, cuya finalidad es resolver una situación determinada con el planteamiento de una solución puntual para el espacio definido dentro de la problemática, en términos de objeto - producto o sistema de objetos - productos, desde cualquier ámbito de la disciplina. Esta modalidad representa el mayor porcentaje de elección de los estudiantes de diseño industrial, puesto que constituye la aplicación de los principios del diseño como actividad proyectual y representa el quehacer del diseñador industrial, con base en los distintos escenarios de la profesión. En segundo lugar, está la pasantía con el $28 \%$ de participación, que se establece como un trabajo de aplicación de los conocimientos teóricos y prácticos del diseño en contextos empresariales específicos, donde el trabajo del diseñador industrial tenga lugar. Esta opción es tomada por los estudiantes, con el propósito de generar espacios laborales que puedan aprovecharse posterior a la titulación. 


\section{Figura 1}

Trabajos de grado por modalidad programa de Diseño Industrial 2000 - 2008

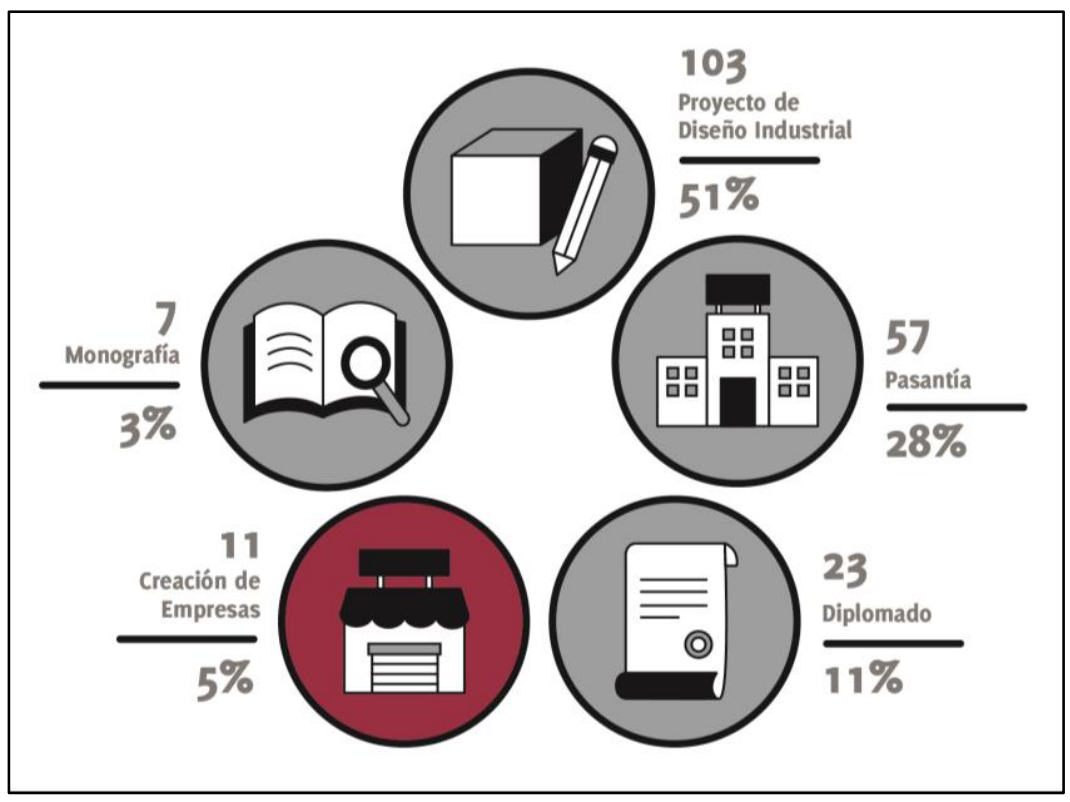

Fuente: elaboración propia.

En tercera instancia con el $11 \%$ se encuentra la modalidad de diplomado, abordado como un curso de formación dentro de un área específica del diseño, que tiene un resultado en términos de proyección y que por su intensidad horaria se identifica como tal. Los diplomados como opción de grado, han facilitado la consecución de la titulación exitosa principalmente para aquellos egresados con situaciones particulares, específicamente derivadas de compromisos laborales. La modalidad de creación de empresas ocupa el cuarto lugar con el 5\% de trabajos de grado y se define como la proyección de un negocio donde el diseño, en cualquiera de sus campos, sea el eje de principal de la actividad desarrollada por la empresa. Esta modalidad, ha sido adoptada por aquellos estudiantes que tienen intereses particulares en algún ámbito específico del diseño industrial, con miras a convertirlo en proyecto productivo, a partir de las posibilidades existentes para el desarrollo de la actividad emprendedora en el contexto del diseño. Por último, se encuentra el proyecto de investigación monografía con el 3\%, que consiste en el desarrollo de un trabajo monográfico escrito, a través del cual se resuelva de manera clara, coherente y profunda, un problema de investigación metodológico, conceptual, productivo, entre otros. Esta modalidad es la que ha tenido menos acogida, por cuanto los desarrollos investigativos tienen unas condiciones específicas como la relación con las líneas de investigación del Departamento de Diseño y además, requieren del acompañamiento de un asesor con 
experiencia en el tema de investigación a abordar. Cabe aclarar, que el panorama de investigativo en el Departamento de Diseño ha cobrado importantes desarrollos desde el año 2016, cuando se abrieron las posibilidades de investigación a los docentes hora cátedra en cuanto al desarrollo de proyectos, participación en grupos de investigación y conformación de semilleros.

\section{Trabajos de grado modalidad creación de empresas}

En cuanto a la modalidad de grado creación de empresas, se han desarrollado 11 proyectos que representan el 5\% de la totalidad de trabajos de grado, con un rango de observación comprendido entre los años 2000 a 2018, siendo el primero Ávalon, mobiliario infantil presentado el 14 de noviembre de 2007 y el último, Llevando los juegos tradicionales al contexto contemporáneo, como herramienta de rescate de tradiciones y costumbres de la región nariñense, presentado el 22 de agosto de 2014 (Tabla 1). Con respecto a las temáticas de los proyectos, cabe destacar que están directamente enfocadas con las asignaturas de Taller de Diseño, cuyo eje principal es la actividad proyectual y así mismo, hay correlación con los talleres de materiales tales como cerámica, maderas, joyería y marroquinería, que están encaminados en el conocimiento, experimentación y materialización por medio tecnologías de transformación, materias primas y procesos de manufactura. Lo anterior evidencia la articulación que deben tener estas dos líneas del área de fundamentación científico tecnológica, con las asignaturas de administración del área de formación complementaria, que hacen parte del plan de estudios del programa de diseño industrial.

\section{Tabla 1}

Trabajos de grado modalidad creación de empresas programa de Diseño Industrial

\begin{tabular}{cll}
\hline FECHA & \multicolumn{1}{c}{ PROYECTO } & \multicolumn{1}{c}{ ESTUDIANTES } \\
\hline $14 / 11 / 2007$ & Ávalon, mobiliario infantil. & Cristina Ascuntar \\
& & \multicolumn{1}{c}{ Claudia Jennifer Leitón } \\
Amanda Taimal \\
$28 / 02 / 2008$ & Khumana, joyería contemporánea. & Fernanda Vallejo \\
$24 / 09 / 2010$ & $\begin{array}{l}\text { Plan de mercado para la creación de una empresa } \\
\text { dedicada al diseño y fabricación de piezas y partes }\end{array}$ & Paola Cupacán \\
& $\begin{array}{l}\text { para vehículos. } \\
19 / 11 / 2010\end{array}$ & Elaboración y comercialización de artículos \\
& conceptuales utilizados en cerámica. & John Jairo Villota \\
\hline
\end{tabular}




\begin{tabular}{|c|c|c|}
\hline $19 / 11 / 2010$ & $\begin{array}{l}\text { Bajo el proceso productivo y técnicas de la cerámica } \\
\text { en la región, generar un plan de negocios que permita } \\
\text { crear empresa desde la perspectiva innovadora del } \\
\text { diseño industrial. }\end{array}$ & $\begin{array}{l}\text { John Alexander } \\
\text { Benavides }\end{array}$ \\
\hline 18/03/2011 & Plup, accesorios convenientes. & Johana Vallejo Benítez \\
\hline $13 / 11 / 2013$ & $\begin{array}{l}\text { Alternativas a objetos comunes de la marroquinería en } \\
\text { Nariño. }\end{array}$ & $\begin{array}{l}\text { David Alejandro } \\
\text { Santacruz }\end{array}$ \\
\hline $15 / 11 / 2013$ & Vicho diseños. & David Andrés Bravo \\
\hline $29 / 05 / 2014$ & $\begin{array}{l}\text { Formulación de un plan de negocios para la ejecución } \\
\text { de una empresa que diseñe, produzca y comercialice } \\
\text { líneas de productos de bisutería. }\end{array}$ & $\begin{array}{l}\text { Fabio Cuastuza } \\
\text { Claudia Figueroa } \\
\text { Carolina Tez }\end{array}$ \\
\hline $19 / 08 / 2014$ & $\begin{array}{l}\text { Plan de negocios para la creación de una empresa } \\
\text { productora y comercializadora para artículos lúdicos } \\
\text { alusivos al carnaval de negros y blancos y para otras } \\
\text { festividades culturales de nuestra nación. }\end{array}$ & $\begin{array}{l}\text { John Robert Rosero } \\
\text { Ordoñez }\end{array}$ \\
\hline $22 / 08 / 2014$ & $\begin{array}{l}\text { Llevando los juegos tradicionales al contexto } \\
\text { contemporáneo como herramienta de rescate de } \\
\text { tradiciones y costumbres de la región nariñense. }\end{array}$ & $\begin{array}{l}\text { José Luis Goyes } \\
\text { Rodríguez } \\
\text { Sergio Ordoñez }\end{array}$ \\
\hline
\end{tabular}

Fuente: elaboración propia.

Cabe anotar que el proyecto Ávalon, mobiliario infantil, de las egresadas Cristina Ascuntar, Claudia Jennifer Leitón, Doris Amanda Taimal y Fernanda Vallejo, obtuvo financiación en la convocatoria del Fondo Emprender del año 2008, hecho que se logró con la asesoría del profesor Julio Garzón quien, para ese entonces, hacía parte de la Unidad de Emprendimiento de la Universidad de Nariño. Igualmente, el proyecto Plup accesorios convenientes de la egresada Johana Vallejo Benítez, obtuvo el beneficio de financiación en la convocatoria del Fondo Emprender del año 2009. Por último, cabe anotar que en el momento de finalización de este estudio en el año 2018, el único emprendimiento que se ha consolidado es Khumana joyería contemporánea, de la egresada Paola Cupacán, empresa que en la actualidad se denomina Cupacán Joyería, y se ha posicionado en el ámbito local y nacional con procesos de gestión, investigación y desarrollo, con recursos económicos propios, estrategias y resultados que le han permitido sostenerse en el mercado de la moda y la joyería. 


\section{Consolidado evaluación de los proyectos}

En referencia a la evaluación de los proyectos de la modalidad de creación de empresas, esta se realiza con base en tres criterios, seis sub-criterios y diez aspectos registrados en el respectivo formato de sustentación (Tabla 2). Dichos criterios están asociados con la forma (informe final), el resultado (prototipo) y sustentación (exposición). En cuanto a los puntajes de los proyectos, la mayor calificación ha sido 96 puntos y la menor 62 puntos, para un promedio de 86,45 en el compendio general de los trabajos de grado. Se destacan dos proyectos que con un puntaje de 96, obtuvieron distinción meritoria y corresponden a Vicho diseños de David Andrés Bravo y Formulación de un plan de negocios para la ejecución de una empresa que diseñe, produzca y comercialice líneas de productos de bisutería de Fabio Cuastuza, Claudia Figueroa y Carolina Tez. Sin embargo, con base en las indagaciones realizadas, esta evaluación no garantiza la continuidad y posterior puesta en marcha del emprendimiento, ya que esta valoración corresponde al proceso académico y por tanto, el compromiso de las partes finaliza con el cumplimiento del requisito de trabajo de grado. Otro aspecto a considerar, es si los criterios de evaluación de los proyectos de esta modalidad, son compatibles con los propósitos, objetivos y resultados que demanda la creación de empresa, por cuanto son aspectos que no necesariamente indagan de manera objetiva sobre el valor de la iniciativa. Sumado a lo anterior, la exigencia en la investigación, el desarrollo del prototipo y la configuración del plan de negocios, implica para el estudiante asumir un proyecto extenso y complejo.

\section{Tabla 2}

Criterios de evaluación para los trabajos de grado modalidad creación de empresas

\begin{tabular}{lll}
\hline \multicolumn{1}{c}{ CRITERIOS } & \multicolumn{1}{c}{ SUB-CRITERIOS } & \multicolumn{1}{c}{ ASPECTOS } \\
\hline Informe final & Contenido & $\begin{array}{l}\text { Coherencia del informe } \\
\text { Proceso de diseño }\end{array}$ \\
& & $\begin{array}{l}\text { Contextualización temática } \\
\text { Coherencia final con el resultado } \\
\text { final }\end{array}$ \\
& Presentación & $\begin{array}{l}\text { Funcionalidad } \\
\text { Integralidad }\end{array}$ \\
& Verificación del resultado & Conceptualización \\
& Objeto de diseño & $\begin{array}{l}\text { Recursos didácticos } \\
\text { Apropiación del tema } \\
\text { Súntesis }\end{array}$ \\
\hline
\end{tabular}

Fuente: elaboración propia. 


\section{Asesorías para la modalidad creación de empresas}

El desarrollo y éxito de los proyectos modalidad creación de empresas, se vincula directamente con la formación y experticia de los docentes asesores en el área de emprendimiento. Con base en el informe de autoevaluación para la Acreditación de Alta Calidad del Programa de Diseño Industrial (Departamento de Diseño, 2019), la formación docente registrada en los últimos cinco años corresponde a las áreas de la gerencia de diseño, el marketing, la experiencia de usuario y la gestión, desarrollo y dirección de proyectos. Dentro de los perfiles no se cuenta de forma explícita el emprendimiento o la creación de negocios como área específica, sin embargo, el talento humano del programa contiene la formación básica para abordar los retos de las fases iniciales del emprendimiento, sobre todo en los aspectos creativos, de Investigación, Desarrollo e Innovación (I+D+I) y aquellos de la especialidad del diseño relacionados con el producto, servicio y experiencia. Con base en la revisión de aspectos metodológicos de los proyectos, cabe mencionar que la implementación del proceso de investigación y de creación de empresas responde esencialmente a las filosofías, métodos y técnicas que el asesor conoce, aplica y confía en su práctica académica y profesional, como es el caso del design thinking, diseño centrado en el usuario DCU, métodos de diseño participativo o de corte proyectual. No obstante, en los referentes conceptuales no se evidencia la implementación de modelos, métodos y técnicas de uso convencional en el proceso emprendedor como el lean startup, lean CANVAS, desarrollo de clientes, propuesta de valor, modelo CANVAS de negocios, plan de negocios, entre otros que, si bien pertenecen a otras necesidades, recursos y contextos, presentan desarrollos y grados de validación que requieren ser adaptados al contexto académico y a la realidad regional. Por otro parte, en el desarrollo de la asesoría, el modelo responde a una intención del programa académico de brindar al estudiante el acompañamiento de un docente para el proceso de investigación orientado a la creación de empresa; no obstante, esta estrategia no es suficiente, puesto que la revisión de las iniciativas demuestra que en el mayor número de los casos, el proyecto de creación de empresas culmina con la obtención del título de diseñador industrial.

\section{Percepciones de los egresados y estado de los proyectos}

De acuerdo con los resultados obtenidos de las entrevistas aplicadas a los egresados, sugieren que la modalidad representa una oportunidad para crear empresa o tener un negocio propio y así mismo, reconocen la necesidad de asumir un proceso formativo que responda a los compromisos que exige la 
modalidad, y que son una serie de entregables que van desde la investigación de necesidades, el prototipo de producto o servicio y finalmente, la configuración del plan de negocios. Cabe advertir que estos tres factores, implican una mayor exigencia en términos investigativos, proyectuales y en el incremento de tiempo y recursos requeridos para abordar cada aspecto. Por otra parte, conviene anotar que en el momento de realización de los proyectos en esta modalidad, el programa de Diseño Industrial no disponía de equipos ni semilleros de investigación, relacionados con modelos de promoción en emprendimientos.

Los entrevistados plantean en prospectiva, que es necesario una reforma del plan de estudios del programa de Diseño Industrial, que fortalezca el emprendimiento y la modalidad de creación de empresas. De la misma forma, sugieren modificaciones en los criterios de evaluación de los trabajos de esta modalidad, que se ajusten a las realidades del proceso de emprendimiento. Según los datos encontrados en registros de procesos de autoevaluación y acreditación del programa (Departamento de Diseño, 2019), entre el año 2001 a 2011, el 19\% de los egresados han creado empresa propia, aunque su iniciativa no haya sido parte de su proyecto de grado. Esto sugiere, la importancia de fortalecer el proceso académico relacionado con la formación de diseñadores industriales emprendedores. Los egresados abordan los servicios profesionales freelance, la creación de negocios y empresas como respuesta a la problemática del desempleo. La necesidad de generar autoempleo y la aspiración de dedicarse a la actividad proyectual en un sector de su interés, surge desde su visión o perspectiva de formación en el estudio y conocimiento del usuario más no de cliente-mercado, que se prevé desde los modelos de pensamiento en emprendimiento. La implementación del enfoque de cliente-mercado en el modelo de pensamiento del diseñador, implica asumir y ajustar los modelos y métodos con enfoque de usuarios.

En cuanto a las motivaciones que tuvieron los egresados para crear su propio negocio, se identific an influencias internas y externas; las primeras emergen del contexto personal y familiar, y las segundas son derivadas de los proyectos de entidades, emprendimientos, empresas de diseño y programas relacionados. Los egresados también reconocen que durante el proceso de formación en emprendimiento encontraron falencias, principalmente en lo que respecta al tema de finanzas, hecho que los condujo a buscar asesoría en otras dependencias constituidas como unidades de emprendimiento. Las percepciones sobre los asesores son diversas y dependen de las expectativas y 
la visión personal de lo que es un emprendedor. Por una parte, los egresados consideran que el asesor debe ser un emprendedor y tener competencias académicas en investigación de mercados, marketing digital y en la construcción del plan de negocios. Otros consideran que no hay claridad de los asesores en el proceso de emprendimiento y perciben la asesoría, más como proceso de diseño con carácter proyectual que con enfoque de creación de empresa. Los egresados manifiestan que acudieron a asesorías fuera del ámbito del programa de Diseño Industrial, donde mencionan la unidad de emprendimiento de la FACEA, como también capacitaciones y asesorías externas recibidas en la Cámara de Comercio de Pasto y el Servicio Nacional de Aprendizaje SENA. La sugerencia de que los asesores sean expertos o con experiencia emprendedora, es precisamente por la inconformidad ante un proceso discontinuo y dispendioso que no corresponde a los resultados de los proyectos de creación de empresas. No obstante, esto no implica que los asesores requieran estas competencias o atributos, por cuanto la demanda es que el diseñador se incorpore en procesos interdisciplinarios, que le permitan responder a las necesidades integrales del proyecto.

En última instancia, es fundamental establecer un diálogo armónico entre administrativos y docentes de las áreas investigativas, proyectuales y de los cursos orientados a la administración, que permita hacer compatibles los propósitos, alcances y compromisos que el programa académico esté dispuesto a asumir, en lo que respecta al fomento y promoción del emprendimiento en diseño.

\section{Conclusiones}

Con base en los resultados obtenidos respecto al desarrollo de trabajos de grado en la modalidad creación de empresas, se evidencia una baja participación que corresponde al $5 \%$ de la totalidad de proyectos de grado. Esta cifra relativamente baja, requiere de atención e indagación sobre el caso particular del programa, en lo que respecta la causalidad y el proceso académico de los estudiantes del programa de diseño industrial. Así mismo, se requiere una toma de conciencia del emprendimie nto como un sistema y un proceso al cual el programa académico está llamado a articularse.

Desde el ámbito académico, es perentorio generar escenarios que estimulen procesos de investigación teóricos, curriculares y pragmáticos, para el fomento y promoción del emprendimiento. En principio, se requiere un mapeo de los emprendimientos de los egresados y determinar los estados de arte, las necesidades y retos a los cuales se ven enfrentados. De esta manera, es posible sopesar las habilidades, 
destrezas y competencias del talento humano del programa en procesos de emprendimiento, donde se debe configurar una relación directa y transversal entre el emprendimiento, el currículo y el plan de estudios, para responder de manera sinérgica a las necesidades y problemas del entorno.

Según el perfil de los docentes quienes fueron asesores de los proyectos modalidad creación de empresas, es perceptible que su formación y competencias corresponden en la actualidad a las áreas de ingeniería multimedia, innovación social, experiencia de usuario, gerencia de diseño, formulación y gestión de proyectos, las cuales responden de forma transversal a aspectos fundamentales del proceso de emprendimiento relacionados con la creatividad e innovación, componentes cruciales en el proceso emprendedor. No obstante, se sugiere realizar la cualificación docente en el área específica de emprendimiento, tanto en investigación teórica y aplicada, como en la cultura y el ecosistema emprendedor, procesos, metodologías, métodos y técnicas propias de esta nueva área de conocimiento.

A manera de síntesis, se hace necesario considerar una reforma a la reglamentación de trabajos de grado en lo referente a la modalidad creación de empresas, ya que actualmente exige labores de investigación, desarrollo de producto y construcción del plan de negocios. Lo anterior, hace que su planteamiento sea complejo y extenso, provocando la desmotivación de los estudiantes para asumir el reto de titularse bajo esta modalidad. Para culminar, la creación de empresas como proyecto de grado, debe estimular al estudiante a abordar la fase de pre-incubación, la idea de negocio y la propuesta de valor, como también la fase de incubación que plantea el modelo y patrón de negocio, realiza ndo actividades de validación con clientes y de mercado.

\section{Referencias}

(1) Alvarado, O. y Rivera, W. (2011). Universidad y emprendimiento, aportes para la formación de profesionales emprendedores. Cuadernos de Administración, 27(45), 61-74.

(2) Arenal, A., Armuña, C., Ramos, S. y Feijóo, C. (2018). Ecosistemas emprendedores y startups, el nuevo protagonismo de las pequeñas organizaciones. Economía Industrial, 85-94. 
(3) Correa, J., Ledesma, J. y Peñaherrera, F. (2019). Importancia de la innovación y creatividad en el desarrollo de productos. Pro Sciences. Revista de Producción, Ciencias e investigación, 3(22), 31-37. https://doi.org/10.29018/issn.2588-1000voBiss22.2019pp31-37

(4) Cuevas, M. F. (2016). Benchmarking de las unidades de emprendimiento en las universidades de Colombia. Questionar: $\quad$ Investigación $\quad$ Específica, 4(1), 109-121. https://doi.org/10.29097/23461098.115

(5) Departamento de Diseño. (2006). Reglamentación de Trabajos de Grado del Programa de Diseño Industrial, Universidad de Nariño. Pasto, Nariño, Colombia.

(6) Departamento de Diseño. (2013). Proyecto Educativo del Programa de Diseño Industrial, Universidad de Nariño. Pasto, Nariño, Colombia.

(7) Departamento de Diseño. (2019). Informe de Autoevaluación para la Acreditación de Alta Calidad - Programa de Diseño Industrial, Universidad de Nariño. Pasto, Nariño, Colombia.

(8) Departamento Nacional de Planeación [DNP]. (2016). Política Nacional de Desarrollo $\begin{array}{llll}\text { Productivo } & \text { (Documento CONPES 3866). DNP. }\end{array}$ https://colaboracion.dnp.gov.co/CDT/Conpes/Econ\%C3\%B3 micos/3866.pdf

(9) Drucker, P. (1994). La innovación y el empresario innovador: La práctica y los principios. EDSA.

(10)Gómez, L., López, S., Hernández, N., Galvis, M., Parra, L., Matiz, F., Varela, R., Moreno, J., Pereira, F., Arias, A., García, G. y Martínez, P. (2019). GEM Colombia: estudio de la actividad empresarial en 2017. Editorial Universidad del Norte. http://manglar.uninorte.edu.co/bitstream/handle/10584/8370/9789587890563\%20eGEM\%20Co lombia\%202017.pdf?sequence $=1 \&$ is Allowed $=\mathrm{y}$

(11) Hurtado, J. (2010). Metodología de la investigación: Guía para la comprensión holística de la ciencia. Quirón Editores.

(12) iNNpulsa Colombia. (2016). Mapeo y caracterización del ecosistema de emprendimiento en Colombia, con énfasis en la localización de las startups. https://innpulsacolombia.com/sites/default/files/documentos-recursospdf/1.2._analisis_y_descripcion.pdf

(13)Lecuona, M. (2006). Manual sobre gestión del diseño para empresas que abren nuevos mercados. Barcelona Centro de Diseño BCD. 
(14)Lederman, D., Messina, J., Pienknagura, S. y Rigolino, J. (2014). El emprendimiento en América Latina: muchas empresas y poca innovación. Banco Mundial. https://oi.org/10.1596/978-14648-0284-3

(15)Ley 344 de 1996. (1996, 27 de diciembre). Congreso de la República de Colombia. Diario Oficial No. 42.951. http://www.secretariasenado.gov.co/senado/basedoc/ley_0344_1996.html

(16)Ley 1014 de 2006. (2006, 26 de enero). Congreso de la República de Colombia. Diario Oficial

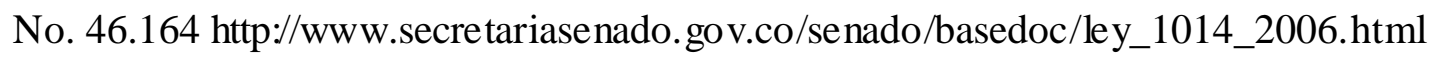

(17)Lora, E., \& Castellani, F. (Eds.). (2014). Entrepreneurship in Latin America: A step up the social ladder. Inter-American Development Bank. http://documents.worldbank.org/curated/en/530831468045044844/pdf/Entrepreneurship-inLatin-America-a-step-up-the-social-ladder.pdf

(18) Mazacón, M., Paliz, C. y Espín, Y. (2019). Emprendimiento en las instituciones de educación superior. Universidad, Ciencia y Tecnología, 2(2), 11-18. https://www.uctune xpo.autanabooks.com/index.php/uct/article/view/212

(19) Minniti, M. (2012). El emprendimiento y el crecimiento económico de las naciones. Economía Industrial, 23-30. https:/pdfs.semanticscholar.org/6ea5/e81d6de26331a9a7729ee77623058c17f655.pdf

(20) Organización de la Cooperación y Desarrollo Económicos [OCDE] y Oficina de Estadísticas de las Comunidades Europeas [EUROSTAT]. (2006). Manual de Oslo (J. Zamorano, Trad.; 3. ${ }^{\mathrm{e}} \mathrm{ed}$.). Grupo Tragsa. (Original work published 2005).

(21)Pulido, S. J., Acosta, M. L., Sánchez, M., Toro, E. y Parra, R. (2017). Didácticas para formar emprendedores. Editorial de Caldas.

(22) Sanguinetti, P., Brassiolo, P., Arreaza, A., Berniell, L., Álvarez, F., Ortega, D. y Kamiya, M. (2013). RED 2013: Emprendimientos en América Latina. Desde la subsistencia hacia la transformación productiva (Reporte de Economía y Desarrollo (RED)). CAF. http://scioteca.caf.com/handle/123456789/168

(23) Schnarch, A. (2014). Emprendimiento exitoso: cómo mejorar su proceso y gestión. ECOE Editores.

(24) Shapero, A., \& Sokol, L. (1982). The social dimensions of entrepreneurship. En C. Kent, D. Sexton and K. Vesper, (Eds.). Encyclopaedia of entrepreneurship (pp. 72-90). 
(25)Suárez, F. (2017). Fomento del emprendimiento a través de actividades académicas e investigativas. Tendencias, 18(2), 183-196. https//doi.org/10.22267/rtend.171802.84

(26) Tarapuez, E., Osorio, H. y Botero, J. J. (2013). Política de emprendimiento en Colombia, 20022010. Estudios gerenciales, 29(128), 274-283. https://doi.org/10.1016/j.estger.2013.09.001

(27)Urbano, D. y Toledano, N. (2008). Invitación al emprendimiento. Una aproximación a la creación de empresas. Editorial UOC.

(28)Uribe, M. y Reinoso, J. F. (2013). Emprendimiento y empresarismo: diferencias, conceptos, cultura emprendedora, idea y proyecto de empresa. Ediciones de la U.

(29) Urrego, A. y Quiroga, A. (2018). Revisión de la literatura respecto a influencia de la educación superior en el emprendimiento, Colombia. https://ciencia.lasalle.edu.co/administracion_de_empresas/1538

(30)World Design Organization [WOD]. (2020, 1 de enero). Definition of Industrial Design. https://wdo.org/

Cómo citar este artículo: Ascuntar, M. y Ayala, F. (2021). Emprendimiento y diseño: caracterización de las iniciativas emprendedoras de los Diseñadores Industriales. Tendencias, 22(1), 95-119. https://doi.org/10.22267/rtend.202102.156 